\title{
Do Tumor Necrosis Factor Inhibitors Cause Malformations in Humans?
}

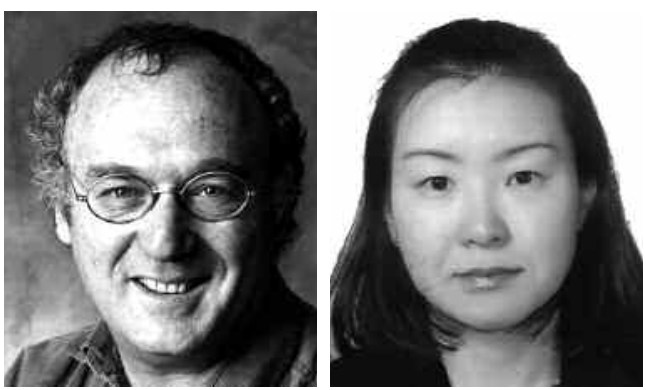

Every year scores of new drugs are introduced with the hope of improving patient care. While all of them are pretested in pregnant animals, no pregnant woman takes them knowingly during premarketing studies, although sometimes pregnancies do occur. Because half of all pregnancies are unplanned, over the life cycle of the drug cases are accumulated of first trimester exposure to new agents ${ }^{1}$. How do we decide whether a drug causes congenital malformations in humans?

Typically, the first line of evidence comes from case reports or case series. Case reports can be very useful, or useless, depending on simple epidemiological reality: If a specific, rare malformation occurs in association with a drug that is taken rarely in pregnancy, then a few case reports may prove causation. For example, the first few cases of anotia (lack of ears) and brain defects after isotretinoin have established causation, because the number of cases by far exceeded the rate of these rare malformations ${ }^{2}$. If the malformation in question is common (e.g., ventricular septal defect), then case reports are useless, as they may merely reflect a chance concurrence. This point will become relevant later, in the context of discussion of tumor necrosis factor (TNF) inhibitors.

The second line of evidence typically comes from cohorts of exposed pregnancies that are compared to a comparison, unexposed group. This design lacks sensitivity, due to the rarity of birth defects in the general population, and therefore often lacks appropriate statistical power. Yet this approach is critical in defining the size of teratogenic risk in absolute terms (i.e., out of 1000 babies how many are likely to have a problem after a specific exposure).

Case-control studies are based on the ability to link specific malformations with gestational exposure to the drug(s) in question. Here, the drug needs to be in use by large populations before it has a chance to be taken in pregnancy. The gold standard of proving causality, the randomized controlled trial, is very rarely performed in the first trimester of pregnancy.
Systematic reviews and metaanalyses are an attempt to combine similar studies to increase their sample size and, hence, increase their ability to detect or reject a "signal" of fetal risk.

In this issue of The Journal, Dr. Carter and colleagues present analysis of the US Food and Drug Administration (FDA) database of spontaneous reporting of adverse events, concluding that TNF antagonists are associated with a potential teratogenic risk in humans ${ }^{3}$.

This specific FDA system collects spontaneous reports, the quality of which is rarely known. Critically, because in this registry all reports are of adverse fetal outcome, Carter and colleagues needed to create a comparison group. To that end they utilized the "normal" or "predictable" distribution of birth defects in the general population. Such an exercise makes the assumption that the spontaneous reports to the FDA can be extrapolated and compared to likelihood of malformation in general. This assumption is tenuous at best as there are many reasons to believe that the spontaneous reports to the FDA have a selection bias, taken from an unknown set of callers to the FDA rather than from the "general population."

For example: the most common finding in Carter's present analysis was cardiac malformations. The most common among cardiac malformations is the muscular-type ventricular septal defect (VSD). In many cases, VSD is spontaneously closed in the first years of life. Hence, depending on when it is detected or reported, the rate will vary dramatically. Women taking TNF antagonists are potentially more likely to have fetal ultrasound and hence, more birth defects may be discovered and reported than among pregnant women in the general population. Moreover, a birth defect in a child with exposure to TNF antagonist in utero may be more likely to be reported to the FDA than the same birth defect in a child with exposure to common drugs ${ }^{4}$.

The futility in establishing causation of human teratogenicity of the spontaneous reporting FDA database was dramatically documented in the case of statins. Similar to

See A safety assessment of TNF antagonists during pregnancy, page 635

Personal non-commercial use only. The Journal of Rheumatology Copyright $@$ 2009 . All rights reserved. 
Carter, et al, Edison and colleagues published a series of cases of malformation after statin exposure, claiming overrepresentation of central nervous system malformation ${ }^{5}$. Alas, subsequently a growing number of cohort studies with hundreds of cases have failed to show any such association ${ }^{6-8}$.

Carter and colleagues perform another unusual scientific leap: they claim that many of the congenital malformations identified in the FDA database associated with TNF inhibitors are "part of" the VACTERL association (vertebral abnormalities, anal atresia, cardiac defect, tracheoesophageal, renal, and limb abnormalities). The VACTERL association is a hodgepodge of different anomalies previously incorrectly attributed to other drugs (e.g., oral contraceptives). Although FDA reports on TNF inhibitors did not confirm diagnoses of VACTERL, Carter, et al suggest that, because they have some features of the VACTERL, the cases ought to be considered as VACTERL.

Despite the very feeble nature of these data, Carter, et al conclude that this purported association "raises concerns of a possible causative effect of the TNF antagonists." For the reasons outlined above, we believe Carter's data are far away from establishing an association, let alone causation.

In the meantime, physicians who will have read the present report may find themselves at unjustified high levels of anxiety ${ }^{1}$. They may choose not to use this class of drug when needed. Even sadder, they may advise women who have been exposed to these drugs inadvertently to terminate otherwise wanted pregnancies. We have shown that women exposed to nonteratogenic drugs believe they have a $25 \%$ risk of major malformations, often leading them to seek termination of otherwise wanted pregnancies ${ }^{9,10}$.

On a grid of 0 to 10 for proving causality, the single case report may score $1-2$, and the randomized controlled trial $8-10$, depending on methodological rigor. On this grid, we believe that the present report scores 1 . Sadly, though, our judgment is likely to be read by selected few, whereas the abstract of this report, which raises "concerns," will be read by thousands, picked up by journalists, and reported by the electronic media. The impact of such tentative signals on women's and physicians' perceptions and decisions have been painfully shown in the case of selective serotonin reuptake inhibitors ${ }^{11}$, where thousands of women discontinued their antidepressants, often after physicians' advice, with worsening of their psychiatric symptoms.

Last, but certainly not least: untreated maternal conditions can gravely increase morbidity and reproductive risks to both mother and unborn children ${ }^{1}$. This must be part of the risk-benefit ratio conducted when evaluating what is the best interest of the mother and her unborn baby ${ }^{12}$.

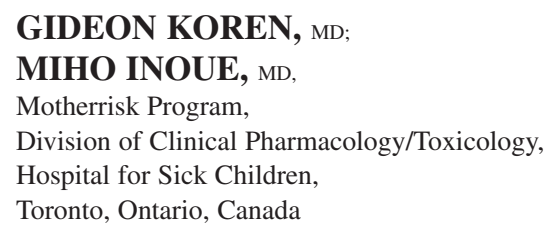

Address reprint requests to Dr. G. Koren, Division of Clinical Pharmacology/Toxicology, Hospital for Sick Children, 555 University Avenue, Toronto, ON, M5G 1X8. E-mail: gkoren@sickkids.ca

\section{REFERENCES}

1. Koren G, Pastuszak A, Ito S. Drugs in pregnancy. N Engl J Med 1998;338:1128-37.

2. Rosa FW. Teratogenicity of isotretinoin. Lancet 1983;2:513.

3. Carter JD, Ladhani A, Ricca LR, Valeriano J, Vasey FB. A safety assessment of TNF antagonists during pregnancy: a review of the FDA database. J Rheumatol 2009;36:635-41.

4. Bar-Oz B, Moretti ME, Mareels G, Van Tittelboom T, Koren G. Reporting bias in retrospective ascertainment of drug-induced embryopathy. Lancet 1999;354:1700-1.

5. Edison RJ, Muenke M. Central nervous system and limb anomalies in case reports of first-trimester statin exposure. $\mathrm{N}$ Engl J Med 2005;352:2759.

6. Pollack PS, Shields KE, Burnett DM, Osborne MJ, Cunningham ML, Stepanavage ME. Pregnancy outcomes after maternal exposure to simvastatin and lovastatin. Birth Defects Res A Clin Mol Teratol 2005;73:888-96.

7. Ofori B, Rey E, Berard A. Risk of congenital anomalies in pregnant users of statin drugs. Br J Clin Pharmacol 2007;64:496-509.

8. Taguchi N, Rubin ET, Hosokawa A, et al. Prenatal exposure to HMG-CoA reductase inhibitors: Effects on fetal and neonatal outcomes. Reprod Toxicol 2008;26:175-7.

9. Koren G, Bologa M, Long D, Feldman Y, Shear NH. Perception of teratogenic risk by pregnant women exposed to drugs and chemicals during the first trimester. Am J Obstet Gynecol 1990;163:695.

10. Pole M, Einarson A, Pairaudeau N, Einarson T, Koren G. Drug labeling and risk perceptions of teratogenicity: a survey of pregnant Canadian women and their health professionals. J Clin Pharmacol 2000;40:573-7.

11. Einarson A, Schachtschneider AK, Halil R, Bollano E, Koren G. SSRI's and other antidepressant use during pregnancy and potential neonatal adverse effects: impact of a public health advisory and subsequent reports in the news media. BMC Pregnancy Childbirth 2005;5:11.

12. Koren G. Medical safety in pregnancy and breastfeeding. New York: McGraw Hill; 2007.

J Rheumatol 2009;36:465-6; doi:10.3899/jrheum.081083 